\title{
Analysis of Similarities and Differences between Acoustic Emission and Charge Signal Based on Fractal Characteristics of Coal Fracture
}

\author{
Xin Ding $\mathbb{D},{ }^{1}$ Xiao-Chun Xiao $\mathbb{D}^{1},{ }^{1}$ Xiang-Feng $L v \mathbb{D}^{2},{ }^{2}$ Di Wu, ${ }^{1}$ and Yishan Pan $\mathbb{D}^{1,3}$ \\ ${ }^{1}$ School of Mechanics and Engineering, Liaoning Technical University, Fuxin 123000, China \\ ${ }^{2}$ School of Civil and Resource Engineering, University of Science \& Technology Beijing, Beijing 100083, China \\ ${ }^{3}$ Environmental Engineering College, Liaoning University, Shenyang 110136, China
}

Correspondence should be addressed to Xin Ding; dingx9054@163.com, Xiao-Chun Xiao; xxc7902@163.com, and Xiang-Feng Lv; lvxiangfeng2006@126.com

Received 20 August 2019; Accepted 6 February 2020; Published 2 April 2020

Academic Editor: Abdul Aziz Bin Abdul Samad

Copyright (c) 2020 Xin Ding et al. This is an open access article distributed under the Creative Commons Attribution License, which permits unrestricted use, distribution, and reproduction in any medium, provided the original work is properly cited.

\begin{abstract}
Rock burst is a catastrophic dynamic disaster caused by sudden failure and instability of coal, which brings threats to deep coal mining; the AE-charge signals and the fragment distribution are related to both mechanical properties of coal and disaster early warning directly. Hence, the variation of $\mathrm{AE}$ and charge induction during coal failure, fractal feature of coal fragments, and their relationship should be studied in depth. In this paper, uniaxial loading test was carried out for coal with bursting tendency samples produced by blocks cored from $800 \mathrm{~m}$ depth in Xiaoqing coal mine of the Tiefa Coal Group in northeast China; the fractal characteristics of specimens are obtained by using the statistical fractal method. The mechanics of similarities and differences between acoustic emission and charge signal is investigated by using loading experiments and theoretical analysis. It is found that the fragments of coal have good self-similarity properties; the fractal dimension of the specimens is in the range 2.085-2.521, the maximum range being 2.300-2.468, which is slightly higher than that of rock. The high-amplitude pulses of the acoustic emission and charge are concentrated in the macroscopic fissure development and expansion stage but they have asynchronous characteristics between them. The charge generation process is accompanied by the inhomogeneous deformation and sliding friction; the friction slip is the major one and is analysed theoretically. A theoretical model for the force-electric coupling relationship is established. The statistical results show that both the acoustic emission and the charge signal accumulation have a significantly proportional relationship with the fractal dimension. Both the acoustic emission and charge signal reveal coal breakage evolution process, which will help in obtaining the precursor information on coal failure. Furthermore, the monitoring results can predict the extent of coal mass instability.
\end{abstract}

\section{Introduction}

Coal is a strong nonlinear engineering rock $[1,2]$, prone to instability and induced dynamic disasters such as rock burst [3-5]. Synchronous monitoring could reveal the trend of stress and strain energy in coal mass and warn of the impending disaster, thus reducing the risk evaluation error efficiently compared with the traditional mechanical-parameters index hazard identification, which is due to the discrete coal mechanical behaviour caused by external load and the internal structure. Therefore, the exploration of effective and reliable monitoring methods has become an engineering challenge in need of an urgent solution for China's coal production industry, predominantly for the purpose of rock burst disaster prevention and control.

It has been widely confirmed by scholars that the sound and electrical anomalies during coal or rock failure process [6-10], which are the acoustic emission or charge signal, can be used for disaster monitoring and early waring [11-13]. In applications, the use of a single monitoring method was limited by important precursor information that was missing, binary signal monitoring of acoustic emission and charge becoming the primary research direction in the disaster monitoring field [14]. During the loading process of 
coal, there are some different manifestations of the two aforementioned parameters, due to the distinct mechanism [15-22]. Therefore, one of the key scientific issues is studying the manifestations and mechanisms of acoustic emission and charge signals, for quantitative analysis of early warning and prediction of dynamic disaster, in overcoming the discrete mechanical behaviour of coal.

Fractal geometry theory describes and studies objective problems based on the concept of fractal dimension and mathematical methods [23], which provide a new way to reveal the complex problems in rock mechanics. Main [24] and Tian et al. [25] explained the mechanism of rock burst using the distribution law of rock fragments and their fractal characteristics. Xie et al. $[26,27]$ introduced fractal theory to the study of rock mechanics for the purpose of simulating the local stochastic irregular roughness on fracture surfaces. $\mathrm{Xu}$ et al. [28] and Liu et al. [29] found that high temperature has a significant effect on the fractal distribution of rock fragments, while the combined effect of high temperature and impact load results in differences in the fragment distributions of rocks. Eremin and Makarov [30] found that the amplitudefrequency characteristics reflected the state of the rock mass and might be considered as the fractal characteristics of the fracture process. Özgen Karacan [31] analysed gamma ray and density logs using univariate statistical techniques and fractal statistics for similarity and ordering of the log data in depth. $\mathrm{Li}$ et al. $[32,33]$ discussed the fractal dimension of AE during coal or rock samples failure affected by preexisting flaw of various inclinations and loading path. The fractal dimension can be used as an indicator that reflects the coal fragments distribution, in mechanical hazard exploration in coal, which has a direct connection with coal crushing.

In the present research, the rock burst appeared mostly in the vicinity of the working face $[2,3]$, the results being the fracturing of coal and instantaneous loss of carrying capacity. Furthermore, the acoustic emission and the charge were accompanied by the rupture of coal. The instability of coal and the sound-electrical anomalies have a direct relevance to the crack propagation. The questions that arise are as follows: (1) what is the intrinsic association between the fractal dimension and sound-electricity anomalies? and (2) how to describe the charge initiation mechanism in coal during friction slip? The acoustic emission mechanism is almost clearly understood, but the charge generation mechanism is not; to date, there is no unified understanding of coal charge generation, but it is believed that it is mainly caused by friction slip. Therefore, a study on the formation of crack interface and piezoelectric effect in coal, and the differences and similarities in the specific forms of expression, which can provide strong guidance for the acoustic emission and charge signal integrated in rock burst is important both in theory and in engineering applications.

In this paper, the fractal dimension of coal fragments and the law of acoustic emission and charge signal accompanying coal deformation under uniaxial loading are investigated by using a method involving the combination of piezoelectric experiments and theoretical analysis. The characteristic of charge signal analysis is deeply referenced to the acoustic emission signals, the mechanics of crack slip friction charge is discussed, and then a theoretical model of lattice slip and inhomogeneous deformation-sliding friction charge generation during coal deformation and failure process is established. The relationship between the fractal dimension of coal fragments and the accumulation of acoustic emission-charge is studied statistically. The results provide a reliable theoretical and experimental basis for obtaining precursor information to monitor rock bursts.

\section{Materials and Methods}

2.1. Preparation of Specimens. The coal specimens were obtained from a depth of $800 \mathrm{~m}$ in the Xiaoqing mine of the Tiefa Coal Group in northeast China. Cylindrical specimens measuring $\varphi 50 \mathrm{~mm} \times 100 \mathrm{~mm}$ were prepared, and both ends of each specimen were polished to ensure that the flatness error was less than $\pm 0.02 \mathrm{~mm}$ (Figure 1 ). Affected by the special occurrence state and material composition of coal, as well as the initial damage formed inside, the samples taken from the same block have different characteristics of color uniformity in the macro, which leads to the dispersion of the mechanical properties of coal samples $[34,35]$.

2.2. Experimental System. Tests were conducted at the Liaoning Key Laboratory of mine environment and disaster mechanics, Liaoning Technical University, Fuxin. A TAW2000 digital hydraulic servo test machine was used for the uniaxial compression test, and load and displacement data acquisition processes were used to automatically obtain the measurements. The machine had a compression capacity of $2000 \mathrm{kN}$ with a resolution of the number of impressions as $5 \%$ o (Figure 2(a)).

A USEA-2 AE monitoring system, manufactured by Beijing Soundwel Technology Ltd., was used in this study. This monitoring system was composed of AE transducers, preamplifier, signal acquisition, and processing and recording units (Figure 2(a)). The AE transducers were placed on the surface of coal and the coupling surface was coated with a coupling agent; the sampling frequency was set to $1000 \mathrm{kHz}$, the preamplification was $40 \mathrm{db}$, gain amplification was $20 \mathrm{db}$, and the number of sampling points was 1024 .

The charge signal was obtained using a self-developed charge-signal monitoring system that included a microelectro-sensitive alloy sheet, preamplifier, acquisition instrument, and computer. The charge amplifier was mounted on the cylinder, the alloy sheet had no contact with the coal surface, and we ensured that the distance between them was maintained at $5 \mathrm{~mm}$ in each experiment. The sampling frequency was set to $1000 \mathrm{~Hz}$, and the sampling point was 1024 Figure (3).

2.3. Experimental Scheme. The experiment involved a uniaxial compression test of coal, where the loading rate was set at $0.01 \mathrm{~mm} / \mathrm{s}$ and the following procedure was carried out:

(a) Place the coal specimen between the top and bottom indenters at each coupling surface with an insulating 


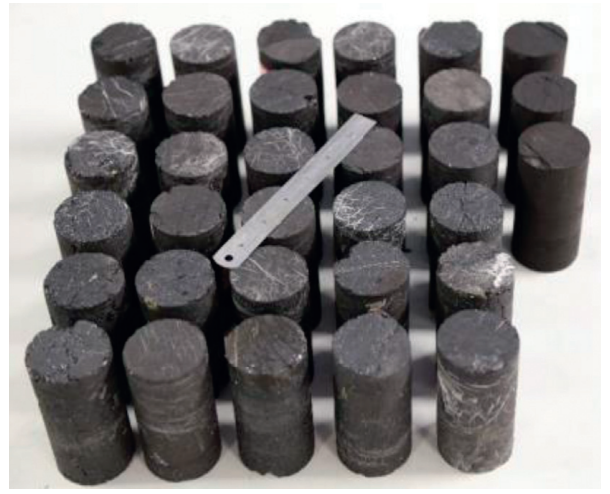

Figure 1: Coal specimens.

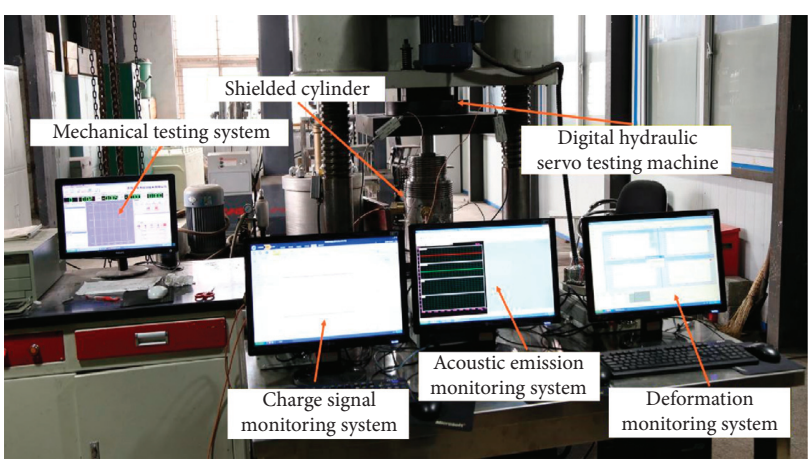

(a)

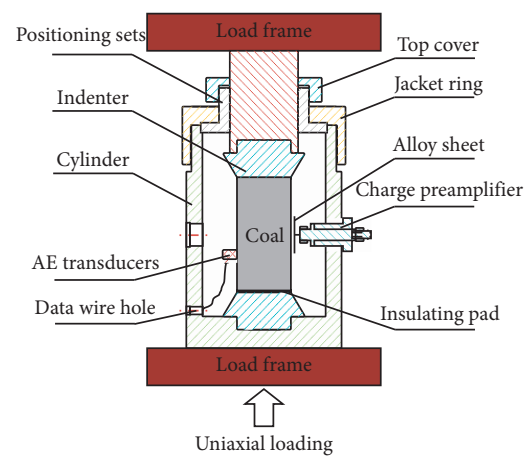

(b)

Figure 2: System for testing. (a) Instruments of the testing system. (b) Structural diagram of the shield cylinder. In the figure, the location of the AE transducers and charge preamplifier is shown, but the number of sensors is not limited.

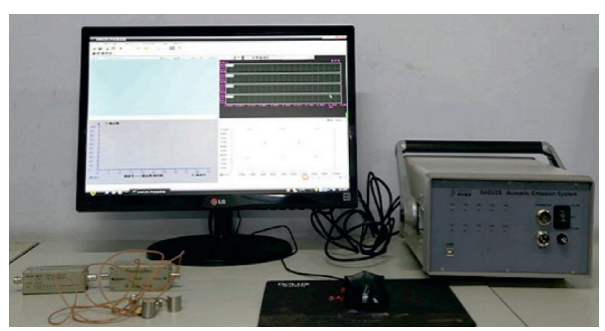

(a)

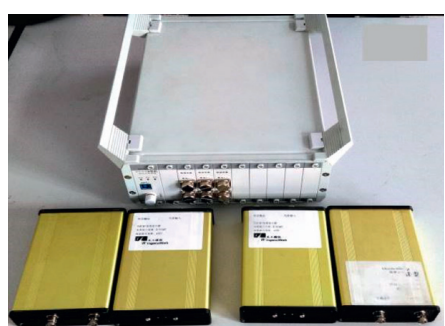

(b)

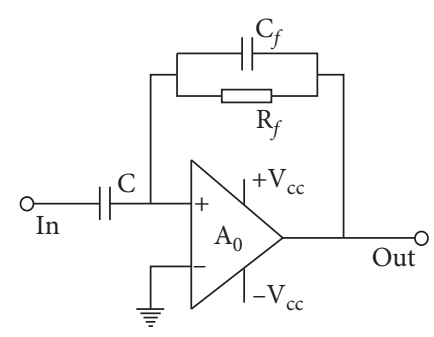

(c)

FIgURE 3: Signal monitoring system. (a) AE monitoring system. preamplifier.

pad to prevent charge overflow, debug equipment, and set parameters.

(b) Start the test system, AE monitoring system, and charge signal monitoring system simultaneously to collect information during the coal deformation and failure process and convert it to a digital signal for storage based on the A/D conversion, until the final destruction. It is worth to point out, where the $\mathrm{AE}$ and charge monitoring system controlled by a synchronization module self-written, to achieve the purpose of simultaneous startup. (c) Store the result, screen the fragments of coal and take photos, and carry out postprocessing of the data on a PC based on Matlab, Origin, or similar software.

2.4. Calculation of the Fractal Dimension of Coal. The results show that the self-similarity of the rock is due to fragments belonging to the statistical fractal distribution [24, 26, 27]; thus the fractal characteristics of coal can be determined by measuring its fragment size and number with the method of screening statistics. However, accurately estimating the 
number of fragments is challenging when the size is less than $5 \mathrm{~mm}$. According to the proportional relationship between mass and block size, $M \propto x^{3}$, coal fragments and fractal dimensions can be calculated using the following massfrequency relationship:

$$
\frac{M(x)}{M_{t}}=\left(\frac{x}{x_{m}}\right)^{3-D_{b}},
$$

where $x$ is the diameter of the fragment, $x_{m}$ is the maximum diameter of a fragment, $M(x)$ is the cumulative quality of fragments with size less than $x, M_{t}$ is total mass of the fragments, and $D_{b}$ is the fractal dimension. Taking the log on both sides of equation (1), we have

$$
\log \frac{M(x)}{M_{t}}=\left(3-D_{b}\right) \log \frac{x}{x_{m}} .
$$

Equation (2) shows that the slope of the fitted straight line is $\left(3 D_{b}\right)$ in the coordinate curve " $\log \left[M(x) / M_{t}\right]-\log \left(x / x_{m}\right)$ "; thus, the fractal dimension of coal fragments can be calculated from the slope of the fitted straight line in the curve, which was under the uniaxial loading.

\section{Result}

3.1. The Characteristics of the Fractal Dimension of Coal Fragments. The broken blocks of coal were collected, and the size distribution of the fragments was obtained by using the method of screening statistics. The sieve size was set as $0-2.5 \mathrm{~mm}, 2.5-5 \mathrm{~mm}, 5-10 \mathrm{~mm}, 10-15 \mathrm{~mm}, 15-20 \mathrm{~mm}$, 20-30 mm, $30-40 \mathrm{~mm}, 40-50 \mathrm{~mm}$, and $50-100 \mathrm{~mm}$. The masses of fragments of each size and the size of the largest fragment and its mass were recorded. Finally, the fractal dimension of each specimen was calculated. Table 1 and Figure 4 present the fragment distribution of coal and the result of fracture. The calculations for the fragment fractal dimension are depicted in Figure 5, which shows coal under uniaxial loading.

The relationship between the mass frequency probability and the cumulative number of pieces is presented in Figure 6. The distribution of fragments with self-similarity characteristics: the mass of fragments changes linearly with the cumulative increase in the number of pieces. Therefore, the fractal theory can be introduced into the study. The greater the fractal dimension, the more the cumulative number of fragments [27]. When the quality and volume of specimens are constant, the cumulative number of fragments is more, and the higher is the degree of fragmentation. In this work, we set the fractal dimension as an indicator of the degree of fragmentation. The fractal dimension of specimens was in the range of 2.085-2.521, mostly within the range of 2.300-2.468, which was slightly higher than that of the rock in the range of 1.7-2.0 [27], which was under the uniaxial loading.

3.2. Results of the Similarity of Acoustic Emission and Charge Signals during the Failure Process of Coal. A set of data results from the experiments was analysed and found to be consistent. Figure 7 indicates the relationship between stress, acoustic emission, and charge signal with time.

In Figure 7, the pink line denotes the charge pulse, the blue scatter is AE energy, and the black line is the stress. The whole loading process of coal deformation is analysed. A set of data results is analysed for the experimental results that are consistent that notable law of acoustic emission and charge signal existed, even though the fractal dimension of coal is different. The loading of coal is divided into three stages: original crack closure, microcrack forming-extending, and macrocrack extending, which is based on the distribution of the signal. In the original crack closure stage, there was some weak acoustic emission signal at first and a gradual decline caused by the original fracture gradually closed with the increase in loading, but no charge pulse appearance. In the microcrack forming-extending stage, there is a significant energy release, but the amplitude of AE is generally in a lower range of $1.0 \times 10^{4} \mathrm{mV} / \mu \mathrm{s}$, indicating that the microcracks have been gradually sprouting and slowly expanding; the weak energy is released to the exterior, and the charge signal is expressed as a sporadic pulse (in Figures $7(\mathrm{a})$ and $7(\mathrm{c})$ ) or no pulse appearance (in Figures $7(\mathrm{~b})$ and $7(\mathrm{~d})$ ). In the macrocrack extended stage, the high-amplitude signal of AE density appeared continuously, and its average value was generally greater than $1.0 \times 10^{4} \mathrm{mV} / \mu \mathrm{s}$. Until the loading reached the first peak stress, the high-amplitude charge signal appeared continuously, and then for both the acoustic emission and charge signal there was a high-amplitude pulse at each stress drop.

To assess the asynchronous phenomenon of $\mathrm{AE}$ and charge signal and its primary reason, the accumulation of $\mathrm{AE}$ energy and charge pulse with time is visualized in Figure 8, each specimen having a different fractal dimension.

There still is a significant consistency law for AE energy and charge generation. It should be noted that the distribution characteristics of the development stage of AE energy and charge accumulation are very similar for the results listed as before. The accumulation of AE energy for 3\%$8.22 \%$ of the total and for the charge was less than $1 \%$, from the original crack stage to microcrack forming-extending. The main part of the AE and charge is generated during the macrocrack extending stage, where the curves of accumulation manifest growth, according to the research result that both of them are directly related to cracks. Therefore, the greater the physical signal generated in this process, the more the number of cracks formed inside the coal.

\subsection{Results of the Difference in Acoustic Emission and Charge} Signals during the Failure Process of Coal. Figure 8 indicates the result of the AE energy and charge pulse evolving with stress at different strengthening stages in the coal rock failure experiment. The local data of loading increased before peak strength, and a stress drop after peak was intercepted. The difference between the two signals was analysed.

Figure 9 shows the difference between acoustic emission and charge signal in response to the variation of stress. For the loading before peak strength, as shown in Figure 9(a), the AE signal varies with a stable and 
TABLe 1: Size and quality of the fragments.

\begin{tabular}{|c|c|c|c|c|c|c|c|c|c|c|c|c|}
\hline & $0-2.5$ & $2.5-5$ & $5-10$ & $10-15$ & $15-20$ & $20-30$ & $30-40$ & $40-50$ & $50-100$ & $\begin{array}{l}\text { Maximum diameter }(\mathrm{mm}) \\
\text { and mass }(\mathrm{g})\end{array}$ & Total mass (g) & Fractal dimension \\
\hline M01 & 9.38 & 10.42 & 25.66 & 13.32 & 9.93 & 22.6 & 46.9 & 43.77 & 95.4 & $51-83.29$ & 277.38 & 2.08465 \\
\hline M02 & 15.92 & 12.48 & 23.91 & 7.29 & 0 & 15.87 & 44.33 & 60.65 & 36.81 & $63.02-34.26$ & 217.26 & 2.29279 \\
\hline M03 & 43.84 & 26.09 & 64.51 & 50.76 & 9.32 & 48.37 & 38.2 & 9.59 & 74.36 & $65.1-74.36$ & 290.68 & 2.30991 \\
\hline M04 & 24.2 & 23.5 & 33.34 & 16.42 & 14.42 & 36.97 & 45.37 & 100.38 & 0 & $50-70.66$ & 294.6 & 2.30002 \\
\hline M05 & 41.39 & 24.49 & 36.9 & 22.73 & 11.61 & 8 & 11.26 & 76.66 & 17.79 & $60-17.79$ & 250.38 & 2.39812 \\
\hline M06 & 19.08 & 27.52 & 57.09 & 29.83 & 7.73 & 40.75 & 17.49 & 29.82 & 0 & $45.56-14.85$ & 229.31 & 2.32257 \\
\hline M07 & 22 & 27.04 & 37.16 & 20.76 & 2.02 & 14.36 & 32.73 & 87.42 & 0 & $50-39.34$ & 243.49 & 2.36342 \\
\hline M08 & 21.65 & 26.49 & 47.64 & 30.75 & 3.72 & 28.1 & 34.62 & 10.86 & 33.72 & $50-33.72$ & 237.55 & 2.35924 \\
\hline M09 & 12.04 & 11.82 & 26.57 & 20.39 & 19.1 & 26.1 & 0 & 53.87 & 65.59 & $74.95-65.59$ & 235.48 & 2.19368 \\
\hline M10 & 12.78 & 10.33 & 10.53 & 5.99 & 0 & 2.57 & 0 & 12.69 & 236.5 & $79.5-100.04$ & 291.39 & 2.36636 \\
\hline M11 & 22.12 & 10.03 & 11.24 & 13.22 & 2.14 & 3.52 & 10.46 & 28.35 & 222.53 & $68.9-104.28$ & 323.61 & 2.39332 \\
\hline M12 & 20.35 & 13.6 & 18 & 2.98 & 1.61 & 9.56 & 13.28 & 19.8 & 159.74 & $85.8-108.68$ & 258.92 & 2.42207 \\
\hline M13 & 7.19 & 6.59 & 9.28 & 9.68 & 1.52 & 6.92 & 2.98 & 52.64 & 182.67 & $98.9-182.67$ & 279.47 & 2.12686 \\
\hline M14 & 17.68 & 18.71 & 30.5 & 22.55 & 2.46 & 38.22 & 36.11 & 18.13 & 71.01 & $63.28-23.28$ & 255.37 & 2.28294 \\
\hline M15 & 23.1 & 14.42 & 8.75 & 2.2 & 0 & 4.28 & 6.94 & 38.61 & 218.9 & $67.20-150.58$ & 317.22 & 2.45008 \\
\hline M16 & 18.69 & 22.2 & 26.05 & 15.91 & 0 & 0 & 13.35 & 26.23 & 141.01 & $62.1-82.38$ & 263.44 & 2.40037 \\
\hline M17 & 24.27 & 17.69 & 18.33 & 4.8 & 3.2 & 6.39 & 15.3 & 23.35 & 164.71 & $73.8-38.75$ & 278.04 & 2.52142 \\
\hline M18 & 18.51 & 10.16 & 7.98 & 0.93 & 2.49 & 7.29 & 0 & 0 & 260.74 & $91.50-111.61$ & 308.1 & 2.45534 \\
\hline M19 & 23.01 & 13.44 & 12.16 & 9.92 & 5.81 & 8.43 & 18.82 & 33.66 & 155.61 & $81.1-83.48$ & 280.86 & 2.40018 \\
\hline M20 & 29.05 & 24.77 & 15.24 & 8.24 & 0 & 1.22 & 33.42 & 36.57 & 152.4 & $53.98-66.73$ & 300.91 & 2.46801 \\
\hline
\end{tabular}

Due to space limitation, only 20 sets of data have been listed.

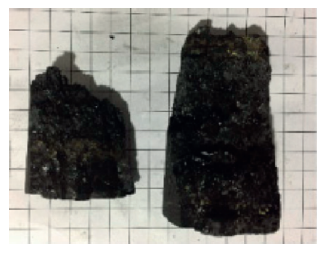

$50-100 \mathrm{~mm}$

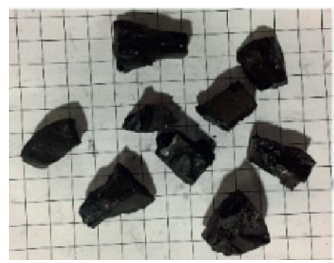

20-30 mm

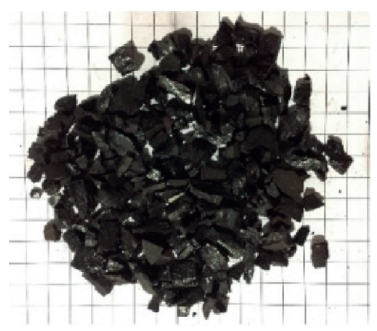

$5-10 \mathrm{~mm}$

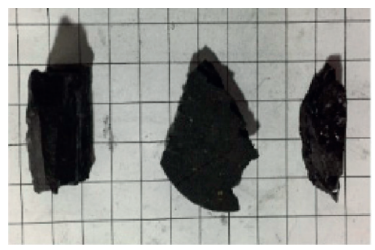

$40-50 \mathrm{~mm}$

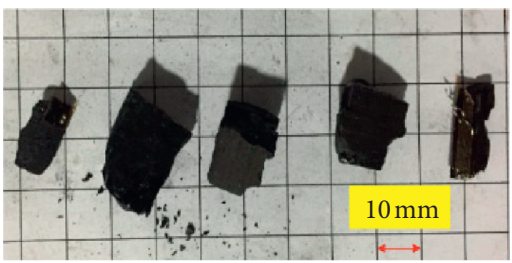

$15-20 \mathrm{~mm}$

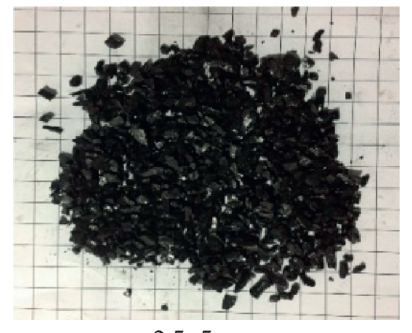

$2.5-5 \mathrm{~mm}$

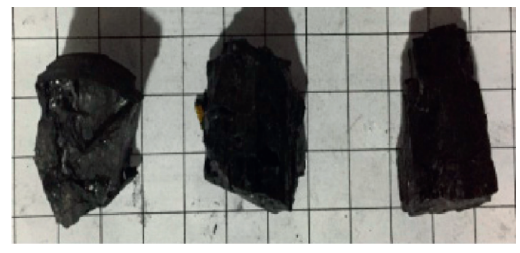

$30-40 \mathrm{~mm}$

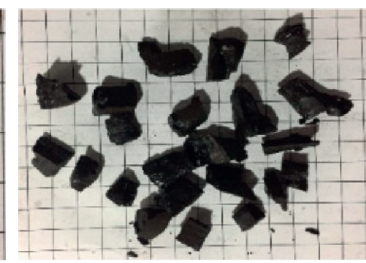

$10-15 \mathrm{~mm}$

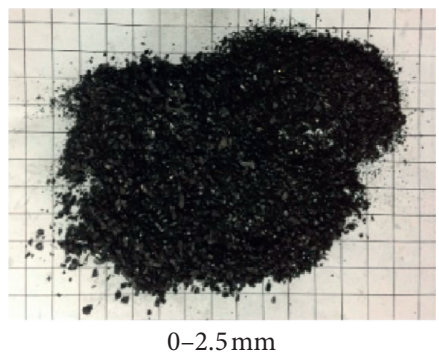

FIgURE 4: Distribution of coal fragments.

continuous high-value signal reflecting the stress increase, but the charge is weak, and the two signals exhibit significant asynchronous features. On the other hand, for the loading after peak strength, as shown in Figure 9(b), the AE and charge signal both produce a significant high-value signal response at the stress drop. The charge amplitude is much higher than that before peak strength but the $\mathrm{AE}$ signal is similar to the size before. This indicates that the $\mathrm{AE}$ signal produced with the nonuniform deformation of coal matrix particles forms microcracks before the peak and the macrocrack expansion at the post-peak strength. The charge generated by the deformation of the coal matrix due to piezoelectric effect before peak and the fracture slip caused by stress drop at the post-peak strength indicates that the charge generated by the fracture slip is much more intense than that by the former. 


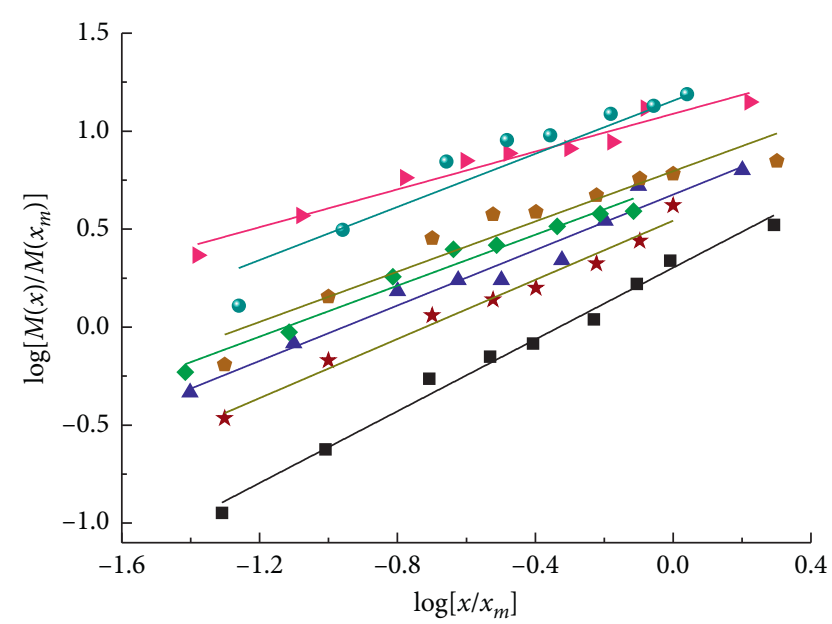

Figure 5: $\log \left[M(x) / M_{t}\right]-\log \left(x / x_{m}\right)$ curve of coal fragments. In the figure, the scattered points are measured results, and straight lines are linear fitted results.

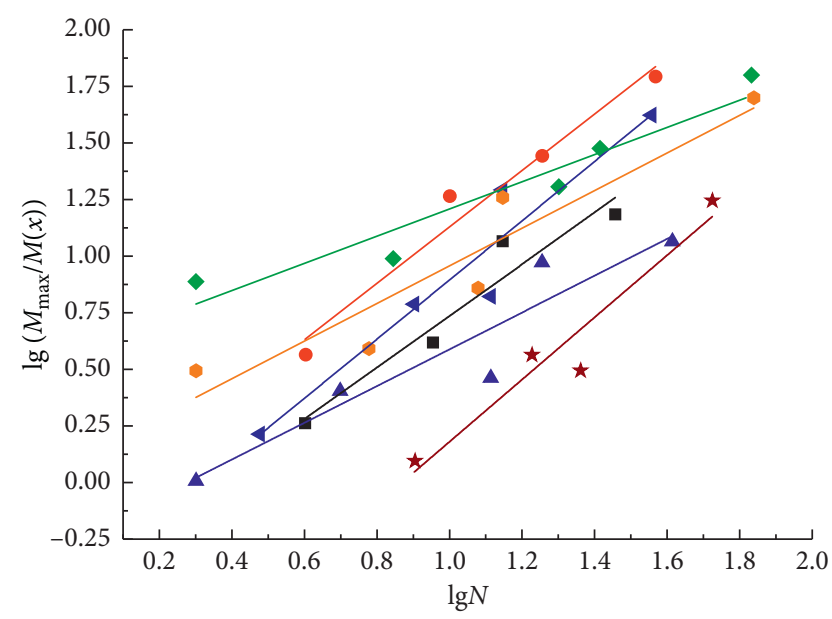

Figure 6: $\lg \left[M(x) / M_{t}\right]-\lg N$ curve of coal fragments. In the figure, the scattered points are measured results, and straight lines are linear fitted results.

Figure 10 displays the result of accumulation of $\mathrm{AE}$ energy and charge with time, with the data of the strength stage before peak strength and stress drop after peak.

We observed that the accumulation of AE energy increased at an approximately linear trend with stress growth. From Figure $10(\mathrm{a})$, the ratio is about $2.5 \times 10^{6} \mathrm{mV} \cdot \mu \mathrm{s}^{-1}$ / $\mathrm{MPa}$, the line of charge shows a ladder-like growth, and its rate is $8.2 \mathrm{pC} / \mathrm{MPa}$ for the loading before peak strength. By contrast, in Figure 10(b), at the post-peak destruction phase, both of them show an improvement in the growth rate. The AE energy still increases linearly, the rate is improved to $4.02 \times 10^{6} \mathrm{mV} \cdot \mu \mathrm{s}^{-1} / \mathrm{MPa}$, and the charge improvement reaches $125 \mathrm{pC} / \mathrm{MPa}$ at the stress drop and little at the "stress platform." The result shows the acoustic emission stable generation with the crack expansion at both the pre- and post-peak strength, but the accumulation rate of the latter is slightly higher than that of the former, while the charge accumulation rate at the post-peak stage is much higher than before, which is especially obvious in the stress drop. This demonstrates that there is a much greater crack development in the posterior peak stage, and the fraction slip between the crack faces is the cause of the majority of the charge.

It is known that the acoustic emission is an elastic wave accompanied by crack propagation; the larger the number of and the faster the expansion of cracks, the more the energy released. In the macrocrack expanding stage, the microcracks gradually turn macroscopic and develop until the complete destruction of coal. The crack propagation in the process of both the before-loading and post-peak strength increases the $\mathrm{AE}$ energy. The degree in damage after-peak strength is higher than that of before-peak strength and the fracture tip stress concentration is much higher than that in the latter. Its propagation speed increases correspondingly and the AE energy grows fast. During the coal failure process, there is not only matrix particle deformation but also large number of crack formations and perforations, according to the research in rock mechanics. Before peak strength, there were a deformation and a small amount of cracks in the coal, while post peak, a large number of expanding macroscopic cracks and a small amount of deformation were observed, with the fissure surface friction at each stress drop. Combined with the test results, we can consider the charge generation during the coal failure to be divided into two stages: the first is before the peak stress expansion process of lattice slip and inhomogeneous deformation, and the second is the post-peak stress process of crack surface friction slip and lattice deformation comprehensive charge initiation, the latter being the foremost reason.

Based on previous research [20], the crystal interface charge generated in the expansion process of inhomogeneous deformation is expressed as

$$
Q=\frac{\varepsilon \bar{t} k T d^{2}}{\delta_{b} S_{b} e \eta \rho}
$$

where $Q$ is the charge number, $\varepsilon$ is the deformation, $S_{b}$ is the diffusion coefficient of the crystal interface, $\delta_{b}$ is the thickness of the crystal interface, $k$ is the Boltzmann constant, $T$ is the absolute temperature, $d$ is the lattice length, and $\eta$ is the resistivity.

The coal loading during post-peak strength includes two parts: crack surface friction slip and lattice deformation. In the stress drop, there was a shear slip friction between both sides of the crack surface. Taking a fracture as the research object, as seen in Figure 10, the sliding friction $\left(F_{d}\right)$ between the two crack surfaces $[36,37]$ can be expressed as

$$
\begin{aligned}
u & =\frac{\mathrm{d} x}{\mathrm{~d} t}, \\
\frac{\mathrm{d} z}{\mathrm{~d} t} & =u-\frac{|u|}{g(u)} z, \\
F_{d} & =\zeta_{0} z+\zeta_{1} \frac{\mathrm{d} z}{\mathrm{~d} t}+\zeta_{2} \frac{\mathrm{d} x}{\mathrm{~d} t},
\end{aligned}
$$




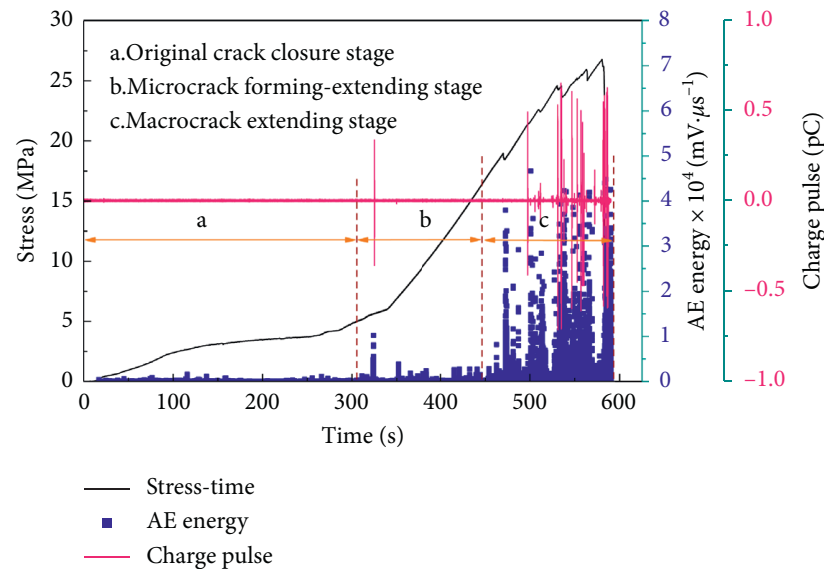

(a)

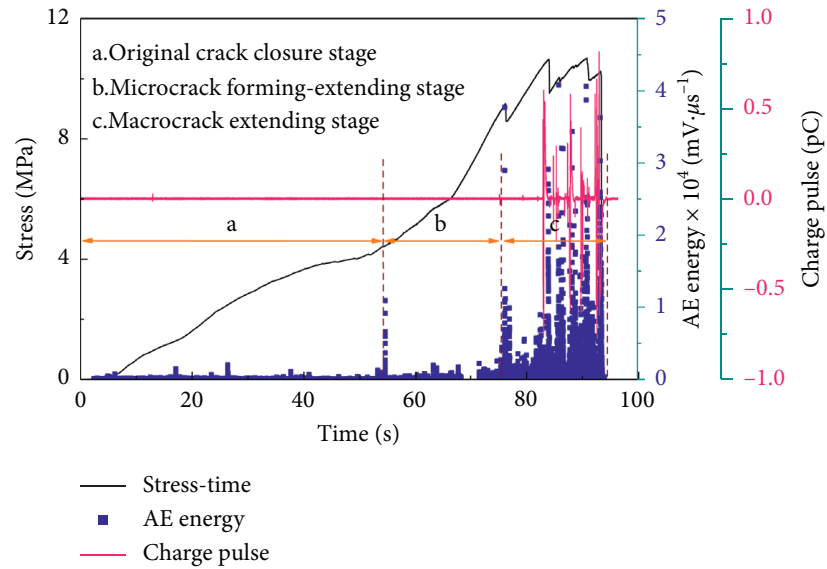

(c)

FIGURE 7: Stress-acoustic emission-charge signal curve of coal.

where $z$ is a state variable, $u$ is the relative velocity between both sides of the crack, $\mathrm{g}(u)$ is a function that models the constant velocity behaviour, $\zeta_{0}$ is an equivalent stiffness for the position-force relationship at velocity reversal, $\zeta_{1}$ is the microviscous friction coefficient, and $\zeta_{2}$ is the viscous friction coefficient.

Figure 11 shows the situation for a crack when it was loaded on post-peak strength. Setting an angle $\theta$ between the fissure extension and the horizontal direction, the falling force $\left(F_{s}\right)$, which causes a shear slip along the upper part of the crack, can be expressed as

$$
F_{s}=\frac{\sigma^{\prime} s \cos \theta}{1-D},
$$

where $\sigma$ is the stress loaded on the crack, $s$ is the loaded area of the fracture, and $D$ is the damage variable.

Based on the friction slip theory [38], the friction slip speed velocity can be expressed as

$$
\frac{\mathrm{d} x}{\mathrm{~d} t}=v-v \cos \left[\sqrt{\left(\frac{K}{m}\right)} t\right]+\frac{F_{s}-F_{d}}{\sqrt{m K}} \sin \left[\sqrt{\left(\frac{K}{m}\right)} t\right],
$$

where $K$ is the string constant, $m$ is the mass of the shear slip part, $t$ is the time, and $v$ is the drive velocity. The loading rate

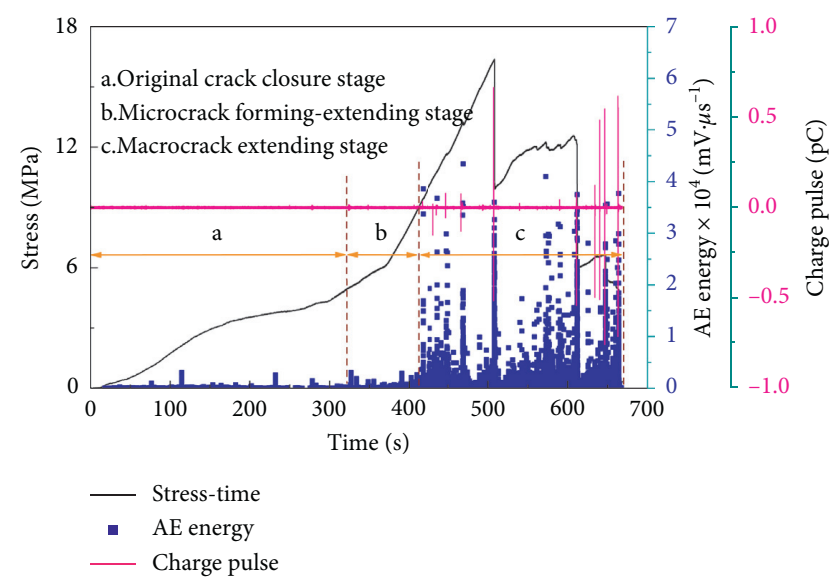

(b)

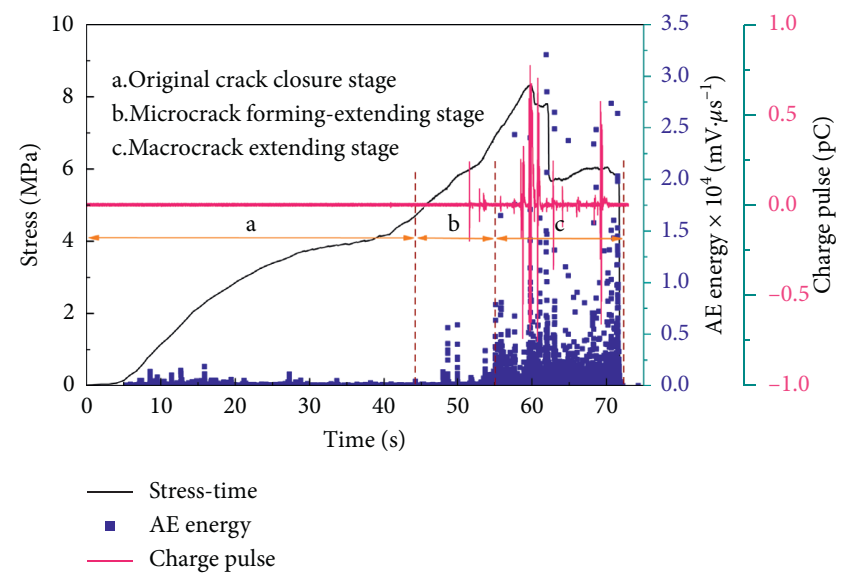

(d)

(a) $D_{b}=2.36342$; (b) $D_{b}=2.19368$; (c) $D_{b}=2.13784 ;$ (d) $D_{b}=2.15006$.

is slower when carried out in the laboratory, and the drive velocity is approximated to $v=0$; then, equation (7) can be written as

$$
\frac{\mathrm{d} x}{\mathrm{~d} t}=\frac{F_{s}-F_{d}}{\sqrt{m K}} \sin \left[\sqrt{\left(\frac{k}{m}\right)} t\right] .
$$

Substituting equations (5) and (6) in equation (8), it can be transformed into

$$
\frac{\mathrm{d} x}{\mathrm{~d} t}=\frac{\left(\left(\sigma^{\prime} s \cos \theta / 1-D\right)-\zeta_{0} z+\zeta_{1}(\mathrm{~d} z / \mathrm{d} t)\right) \sin [\sqrt{(k / m)} t]}{\sqrt{m K}+\zeta_{2} \sin [\sqrt{(k / m)} t]} .
$$

The relative displacement of the two contact surfaces is

$$
x=\int_{0}^{t} \frac{\left(\left(\sigma^{\prime} s \cos \theta / 1-D\right)-\zeta_{0} z+\zeta_{1}(\mathrm{~d} z / \mathrm{d} t)\right) \sin [\sqrt{(k / m)} t]}{\sqrt{m K}+\zeta_{2} \sin [\sqrt{(k / m)} t]} \mathrm{d} t .
$$

Assuming that the post-peak bearing structure is elastomeric, still consistent with Hooke's law, the variation of axial load $(\Delta F)$ caused by the shear slip between the two cracks is given by 


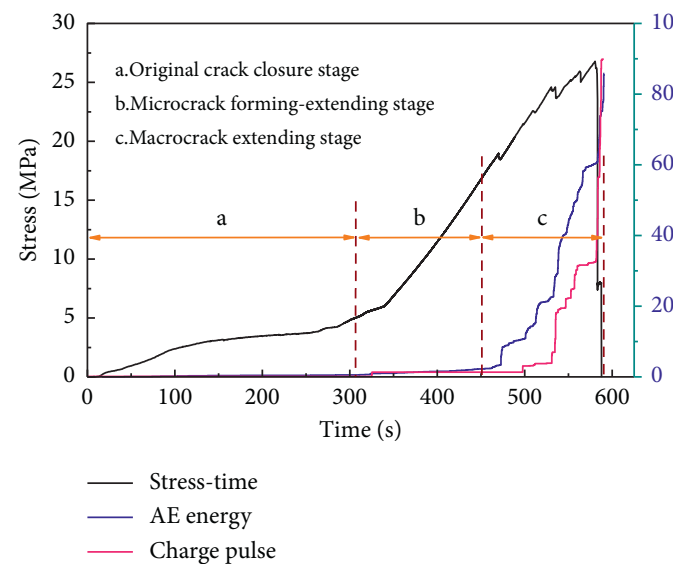

(a)

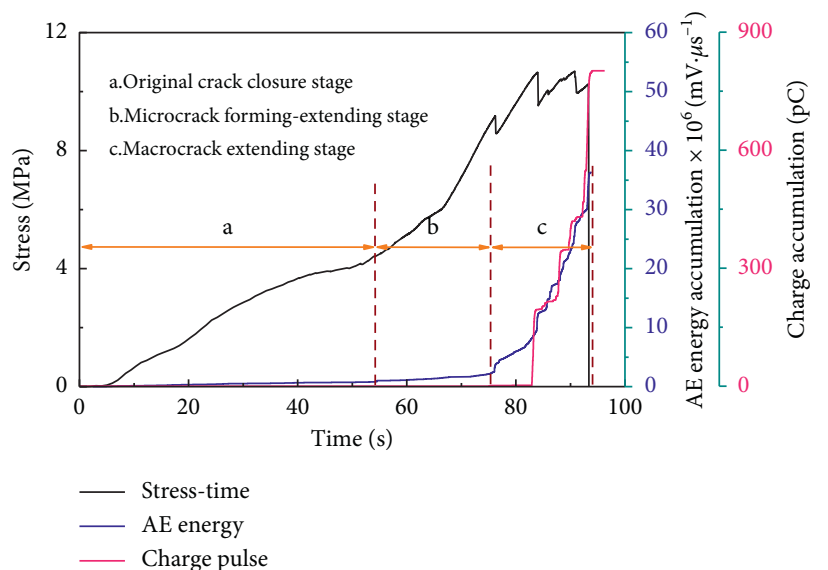

(c)

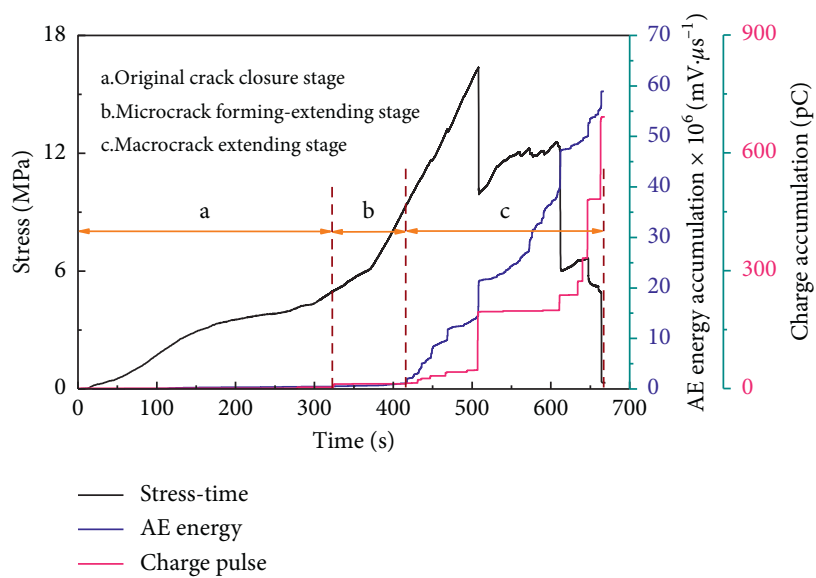

(b)

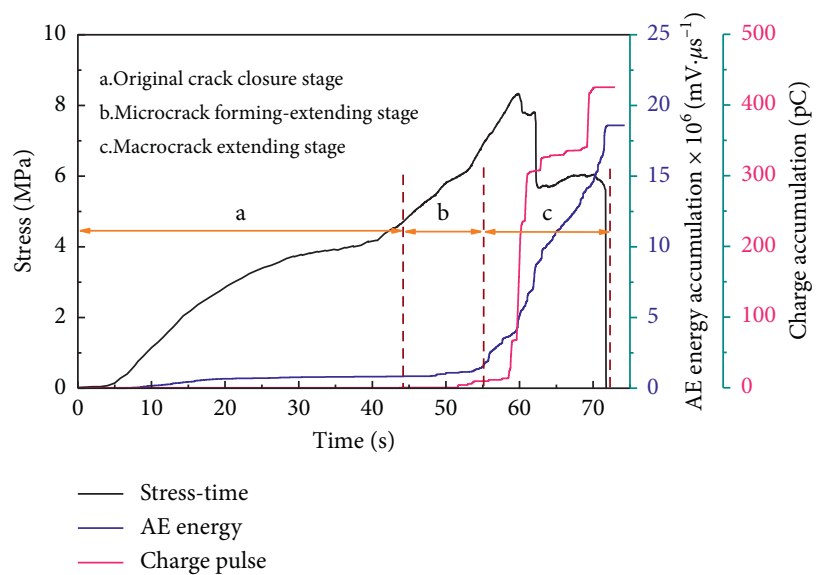

(d)

Figure 8: Changes in the total AE energy-charge accumulation and fractal dimensions. (a) $D_{b}=2.36342$; (b) $D_{b}=2.19368$; (c) $D_{b}=2.13784$; (d) $D_{b}=2.15006$.

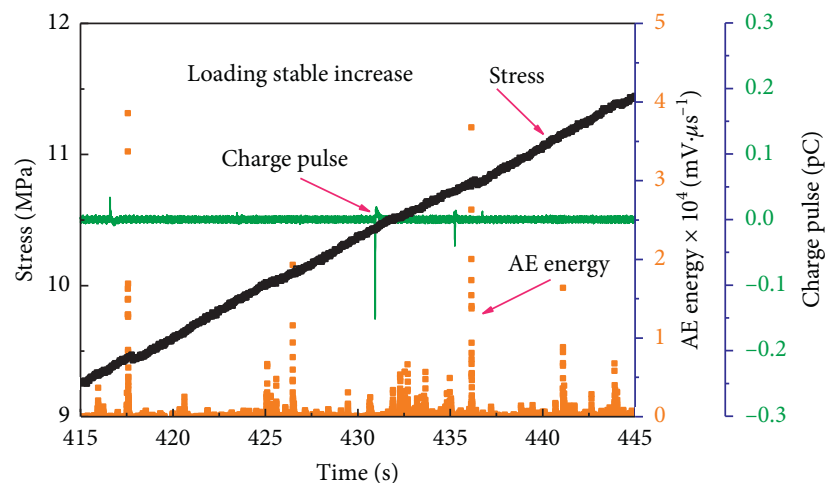

(a)

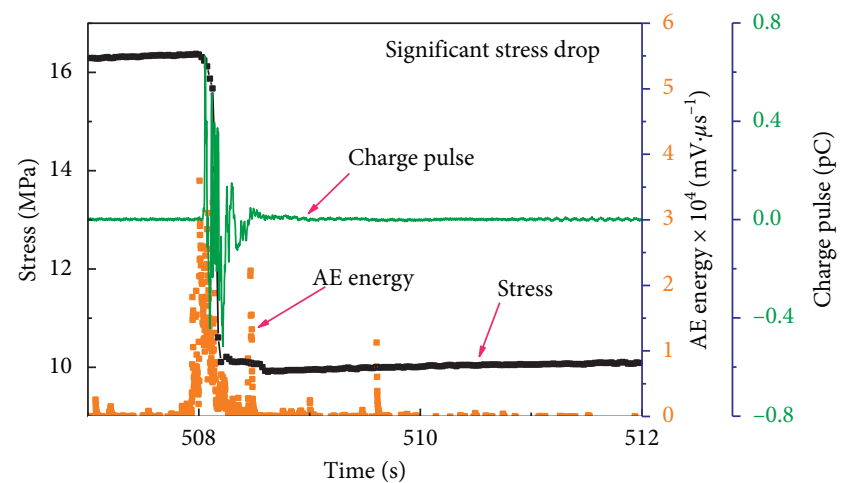

(b)

Figure 9: Evolution of AE energy and charge pulse with stress. (a) The stress growth before peak strength. (b) The stress drop after peak strength.

$$
\Delta F=\frac{x \cdot \cos \theta}{l \cdot(1-D)} E s,
$$

where $l$ is the original length of coal, $E$ is the elastic modulus, and $s$ is the cross-sectional area of coal.
The relative shear slip, which causes friction sliding electricity, the amount of charge, and stress drop are directly proportional [39] and the relationship between them is 


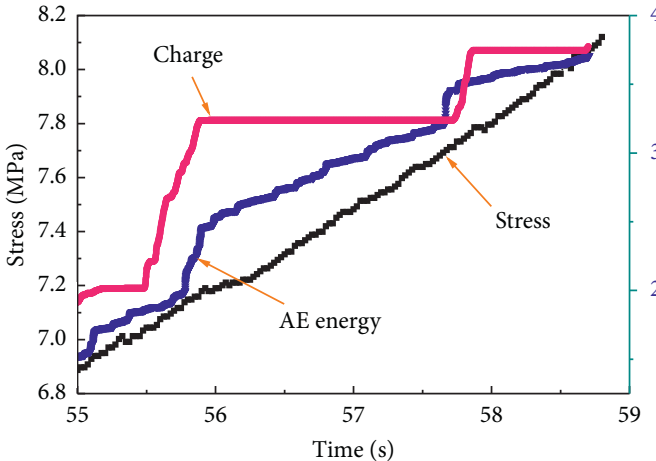

(a)
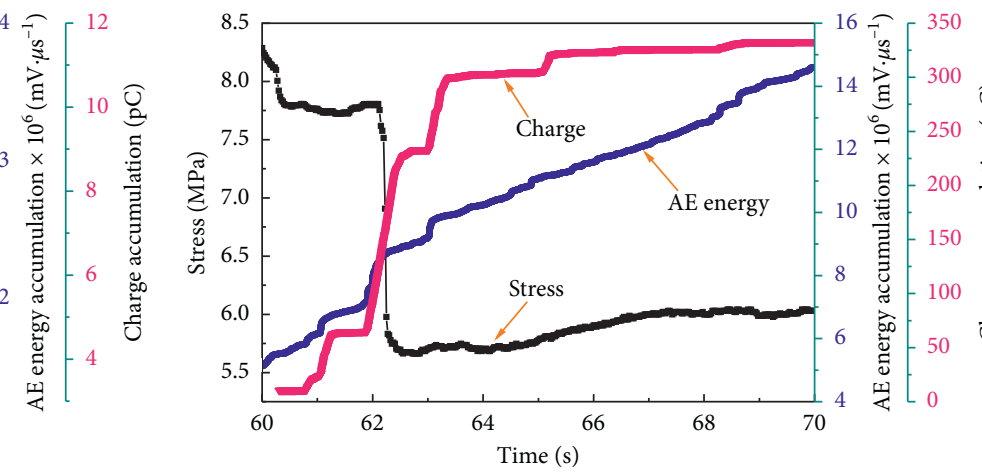

(b)

Figure 10: Accumulation of AE energy and charge evolution with stress. (a) The stress growth before peak strength. (b) The stress drop after peak strength.

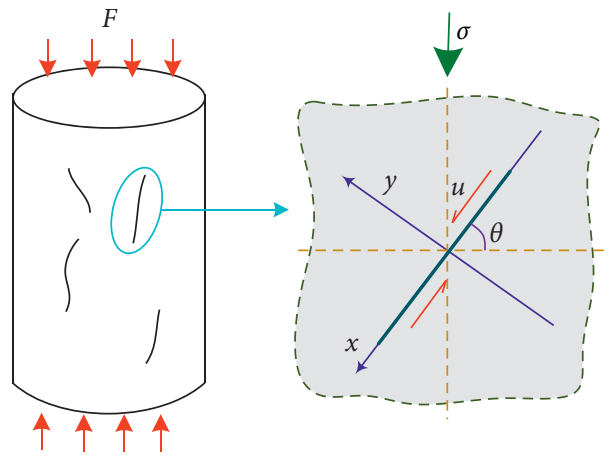

FIGURE 11: Loaded condition for a crack.

$$
n_{q}=\frac{\Delta F}{\alpha}
$$

where $n_{q}$ is the amount of charge generated and $\alpha$ is the average lateral force per charge, related to the electrical property of coal. Combined with equations (10) (12), the model of cracks shear slip friction electoral induction can be defined as follows:

$$
\begin{aligned}
Q= & \frac{e E s \cos \theta}{\alpha l \cdot(1-D)} \\
& \cdot \int_{0}^{t} \frac{\left(\left(\sigma^{\prime} s \cos \theta / 1-D\right)-\zeta_{0} z+\zeta_{1}(\mathrm{~d} z / \mathrm{d} t)\right) \sin [\sqrt{(k / m)} t]}{\sqrt{m K}+\zeta_{2} \sin [\sqrt{(k / m)} t]} \mathrm{d} t .
\end{aligned}
$$

Therefore, combined with equation (3), the model of force-electric coupling of coal charge generated in the failure process, which is the mechanics of inhomogeneous deformation and sliding friction, can be expressed as

$$
Q= \begin{cases}\frac{\varepsilon \bar{t} k T d^{2}}{\delta_{b} S_{b} e \eta \rho}, & 0 \leq \varepsilon \leq \varepsilon_{c}, \\ \frac{\varepsilon \bar{t} k T d^{2}}{\delta_{b} S_{b} e \eta \rho}+\frac{e E s \cos \theta}{\alpha l \cdot(1-D)} \cdot \int_{0}^{t^{\prime}} \frac{\left(\left(\sigma^{\prime} s \cos \theta / 1-D\right)-\zeta_{0} z+\zeta_{1}(\mathrm{~d} z / \mathrm{d} t)\right) \sin [\sqrt{(k / m)} t]}{\sqrt{m K}+\zeta_{2} \sin [\sqrt{(k / m)} t]}, \quad \varepsilon \geq \varepsilon_{c} .\end{cases}
$$




\section{Discussion}

The form of destruction of coal is ever-changing affected by its characteristic of discreteness, noncontinuity, and nonuniformity, which is mainly reflected in the fragment size and mass distribution. This is more obvious in the uniaxial compression process, and then the fractal dimension can be used to describe coal failure. The acoustic emission and charge are accompanied by destruction, and therefore, they are the precursors to the whole process of destruction. Figures 12 and 13 indicate the statistical results of AE energy and charge accumulation of coal specimens with different fractal dimensions, both of them having a linearly positive correlation with the fractal dimension, which indicates that the higher the fractal dimensions, the more the signals generated during the process of coal destruction. A research [1] investigated the relationship between the new surface area formed and the particle size of coal after crushing, as in equations (15) and (16), which indicates that the new surface area is inversely proportional to the weighted average diameter of the fragment; the smaller the particle size, the higher the fractal dimension. Therefore, according to the calculation method for fractal dimension, the higher the fractal dimension of the coal fragments, the larger the new surface area generated:

$$
\begin{aligned}
S & =\frac{60 M_{t}}{\rho d_{a}}, \\
d_{a} & =\frac{\sum_{x=1}^{n} m_{x} \cdot d_{x}}{M_{t}},
\end{aligned}
$$

where $S$ is the new surface area, $M_{t}$ is the total mass of the specimen, $\rho$ is the density of coal, $d_{a}$ is the weighted average diameter of the fragment, $d_{x}$ is diameter of the fragment, and $m_{x}$ is its mass.

A higher fractal dimension of coal fragments means there is more new surface area generated with the coal sample breakdown, which is more native, and new fissures participate in this process. For acoustic emission, there is more crack propagation, implying greater area of crack formed, and it would have released greater elastic performance, more signals will be received by the transducers, and thus, the total AE energy accumulated is mounted with the increase in the fractal dimension. For the charge, according to the previous conclusion, where the friction slip is the major charge generation process in coal, the increase in new surface area generated indicates that more cracks are involved in friction sliding. A large amount of charge is generated with the damage variable of specimen of friction slip at each stress drop. The fractal dimension is the indicator of the damage in the coal; the more the cracks generated due to mutual friction inside the coal, the more the total charge accumulated before the coal sample ruptures. Thus, the charge has a positive correlation with the fractal dimension identical.

Therefore, the precursor information on acoustic emission and charge can be obtained before the coal sample breaks

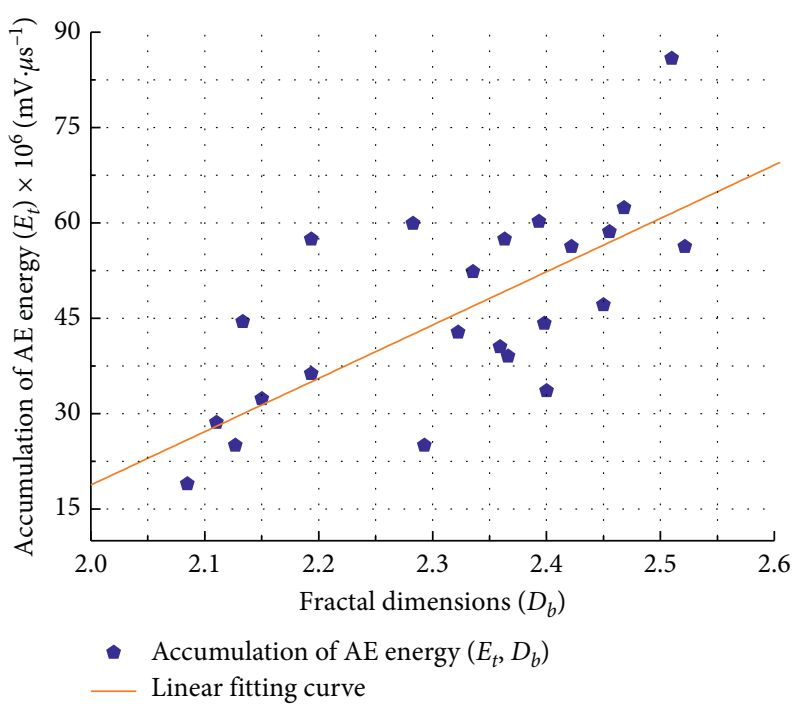

FIgURE 12: Relationship between accumulation of AE energy and fractal dimensions.

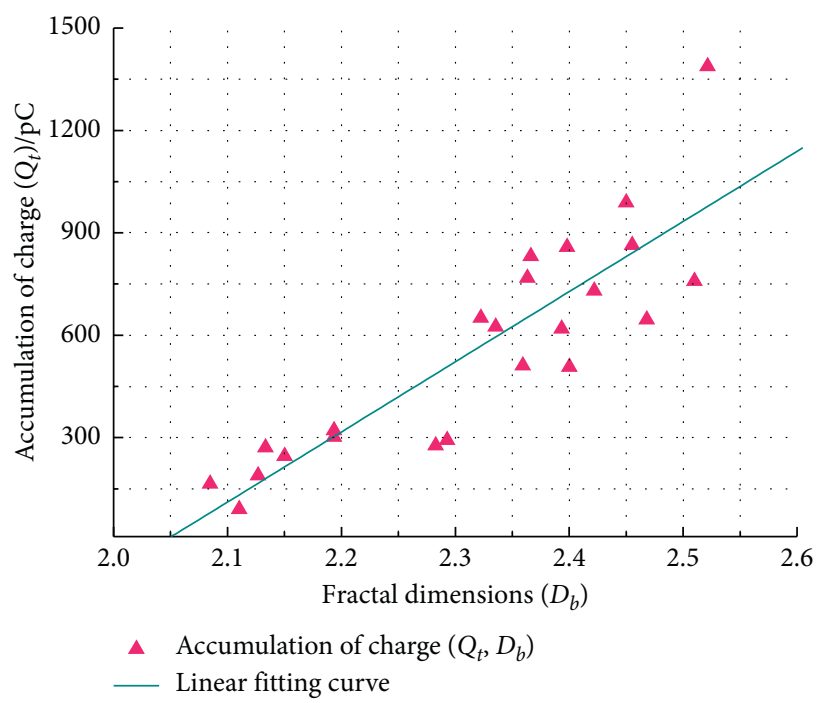

FIGURE 13: Relationship between accumulation of charge and fractal dimensions.

down and can demonstrate the rupture. The monitoring results can predict the extent and state of coal breakage.

\section{Conclusion}

In this paper, a uniaxial compression experiment was carried out to investigate the feature characteristic AE events and charge induction pulse during the failure process of coal samples, the fractal feature of fragments was obtained by the statistical method, and the similarities and differences of acoustic emission and charge signals were theoretically analysed. The following conclusions can be drawn:

(1) The experimental results confirm that coal failure also belonging to the category of statistical fractal research, which the fragments of it after crushing had 
a good self-similarity property under uniaxial loading, can be calculated by mass of different size pieces and total. Therefore, the fractal dimension of the specimens was $2.085-2.521$, with a maximum of $2.300-2.468$, which was slightly higher than that of rock, effected by its special occurrence environment and composition; the larger the fractal dimension, the higher the degree of coal fragmentation, and then the fractal dimension can be used as a mathematical expression describing the coal breakage evolution process.

(2) The acoustic emission and charge signal in the process of coal failure have significant regularity with the loaded stage. The high-amplitude pulse of acoustic emission and charge are concentrated in the macroscopic fissure development and expansion stage, but there are asynchronous characteristics between them. The AE is approximately the same in the pre- and post-peak strength; the charge generated in the former is much more than that in the latter. Based on the cumulative distribution of $\mathrm{AE}$ energy and charge in coal deformation, taking the acoustic emission signal as a reference, we theoretically analysed the charge generation process, which is generated by the inhomogeneous deformation and sliding friction, the friction slip being the major contributor. Then, a theoretical model of the forceelectric coupling relationship was established.

(3) Both the acoustic emission and the charge signal accumulation have a significantly proportional relationship with the fractal dimension. It is shown that the more new fragments generated during the coal failure and the higher the degree of crushing, the richer the acoustic emission events and charge pulse generated in process, and the richness of the signal increases with the deterioration of coal while stress improving. Thus, the precursor information can be obtained before the coal sample breaks down, and demonstrates the rupture. The results provide a theoretical basis for further development of acousticcharge composite signal monitoring.

\section{Data Availability}

The figure data used to support the findings of this study have been deposited in the paper repository $(10.1155 / 2019 /$ 7581061).

\section{Conflicts of Interest}

The authors declare that they have no conflicts of interest.

\section{Acknowledgments}

This work was supported by the National Key Research and Development Program of China (no. 2017YFC0804208) and National Natural Science Foundation of China (nos. 51774164, 51974186, and 51974147).

\section{References}

[1] Z. Meng-Tao, Y.-S. Pan, B. Liang, and L. Wang, Coal and Rock Fluid Mechanics, China Science Publishing \& Media Ltd, Beijing, China, 1995, in Chinese.

[2] Y.-J. Yang, L.-Y. Xing, H.-Q. Duan, L. Deng, and Y.-C. Xue, "Fatigue damage evolution of coal under cyclic loading," Arabian Journal of Geosciences, vol. 11, no. 18, p. 560, 2018.

[3] Y.-S. Pan, L. Zhong-Hua, and M.-T. Zhang, "Distribution, type, mechanism and prevention of rockbrust in China," Chinese Journal of Rock Mechanics and Engineering, vol. 22, no. 11, pp. 1844-1851, 2003, in Chinese.

[4] Y. Jin, G. Liu, Y. Cai, J. Zhou, S. Liu, and B. Tu, "Time-dependent deformation mechanism for swelliing soft-rock tunnels in coal mines and its mathematical deduction," International Journal of Geomechanics, vol. 20, no. 3, Article ID 04019186, 2020.

[5] Bo. Zhao, J. Cao, H. Sun, G. Wen, D. Linchao, and B. Wang, "Experimental investigations of stress-gas pressure evolution rules of coal and gas outburst: a case study in Dingji coal mine China," Energy Science \& Engineering, vol. 8, no. 1, pp. 61-73, 2019.

[6] Y. Enomoto and H. Hashimoto, "Emission of charged particles from indentation fracture of rocks," Nature, vol. 346, no. 6285 , pp. 641-643, 1990.

[7] D. Eccles, P. R. Sammonds, and O. C. Clint, "Laboratory studies of electrical potential during rock failure," International Journal of Rock Mechanics and Mining Sciences, vol. 42, no. 7-8, pp. 933-949, 2005.

[8] V. S. Kuksenko, K. F. Makhmudov, and A. V. Ponomarev, "Relaxation of electric fields induced by mechanical loading in natural dielectrics," Physics of the Solid State, vol. 39, no. 7, pp. 1065-1066, 1997.

[9] Q. Liu, R. Song, J. Liu, Y. Lei, and X. Zhu, "Pore-scale visualization and quantitative analysis of the spontaneous imbibition based on experiments and micro-CT technology in low permeability mixed-wettability rock," Energy Science \& Engineering, pp. 1-17, 2020.

[10] H. Yang, G. Wen, Q. Hu, Y. Li, and L. Dai, "Experimental investigation on influence factors of acoustic emission activity in coal failure process," Energies, vol. 11, no. 6, p. 1414, 2018.

[11] Y. Cai, D. Liu, J. P. Mathews et al., "Permeability evolution in fractured coal-combining triaxial confinement with X-ray computed tomography, acoustic emission and ultrasonic techniques," International Journal of Coal Geology, vol. 122, pp. 91-104, 2014.

[12] M. C. He, J. L. Miao, and J. L. Feng, "Rock burst process of limestone and its acoustic emission characteristics under truetriaxial unloading conditions," International Journal of Rock Mechanics and Mining Sciences, vol. 47, no. 2, pp. 286-298, 2010.

[13] Z. H. Li, E. Y. Wang, X. Y. Song et al., "Study on strain location and surface potential distribution law of coal samples fracturing," Journal of China Coal Society, vol. 37, no. 12, pp. 2043-2047, 2012, in Chinese.

[14] X. Xiao, H. Luo, Y. Pan et al., "Development and application of charge monitoring system of deep mine dynamic disaster," China Safety Science Journal, vol. 24, no. 3, pp. 97-102, 2014, in Chinese.

[15] Y. Zhao and Y. Jiang, "Acoustic emission and thermal infrared precursors associated with bump-prone coal failure," International Journal of Coal Geology, vol. 83, no. 1, pp. 11-20, 2010.

[16] F. T. Freund, A. Takeuchi, and B. W. S. Lau, "Electric currents streaming out of stressed igneous rocks-a step towards 
understanding pre-earthquake low frequency EM emissions," Physics and Chemistry of the Earth, Parts $A / B / C$, vol. 31, no. 4-9, pp. 389-396, 2006.

[17] A. Rabinovitch, D. Bahat, and V. Frid, "Comparison of electromagnetic radiation and acoustic emission in granite fracturing," International Journal of Fracture, vol. 71, no. 2, pp. R33-R41, 1995.

[18] I. Yamada, K. Masuda, and H. Mizutani, "Electromagnetic and acoustic emission associated with rock fracture," Physics of the Earth and Planetary Interiors, vol. 57, no. 1-2, pp. 157-168, 1989.

[19] X. Xiao-Chun, X. Ding, Y.-S. Pan, X. Wang, J. Xu, and H. Luo, "Experiment of acoustic emission and charge induction in granular coal rock failure," Journal of China Coal Society, vol. 40, no. 8, pp. 1796-1804, 2015, in Chinese.

[20] X. Lv, Y. Pan, X. Xiao, and A. Wang, "Barrier formation of micro-crack interface and piezoelectric effect in coal and rock masses," International Journal of Rock Mechanics and Mining Sciences, vol. 64, pp. 1-5, 2013.

[21] J. W. Archer, M. R. Dobbs, A. Aydin, H. J. Reeves, and R. J. Prance, "Measurement and correlation of acoustic emissions and pressure stimulated voltages in rock using an electric potential sensor," International Journal of Rock Mechanics and Mining Sciences, vol. 89, pp. 26-33, 2016.

[22] E. D. Pasiou and D. Triantis, "Correlation between the electric and acoustic signals emitted during compression of brittle materials," Frattura Ed Integrità Strutturale, vol. 11, no. 40, pp. 41-51, 2017.

[23] B. B. Mandelbrot, The Fractal Geometry of Nature, W. H. Freeman, New York, NY, USA, 1982.

[24] I. G. Main, "A modified Griffith criterion for the evolution of damage with a fractal distribution of crack lengths: application to seismic event rates and b-values," Geophysical Journal International, vol. 107, no. 2, pp. 353-362, 1991.

[25] B.-Z. Tian, S.-J. Liu, Y.-B. Zhang, and Z.-L. Wang, "Analysis of fractal characteristic of fragment from rock burst tests under different loading rates," Tehnicki vjesnik-Technical Gazette, vol. 23, no. 5, pp. 1269-1276, 2016.

[26] H. P. Xie, Fractal in Rock Mechanics, A. A. Balema Publishers, Rotterdam, Netherlands, 1993.

[27] H. Xie, H. Sun, Y. Ju, and Z. Feng, "Study on generation of rock fracture surfaces by using fractal interpolation," International Journal of Solids and Structures, vol. 38, no. 32-33, pp. 5765-5787, 2001.

[28] X. L. Xu and Z. Z. Zhang, "Fractal characteristics of rock fracture surface under triaxial compression after high temperature," Advances in Materials Science and Engineering, vol. 2016, Article ID 2181438, 10 pages, 2016.

[29] S. Liu and J. Y. Xu, "Fractal analysis for dynamic failure characteristics of granite induced by mechanical-thermal loading," Géotechnique Letters, vol. 5, no. 3, pp. 191-197, 2016.

[30] M. O. Eremin and P. V. Makarov, "Fractal characteristics of seismic process in rock mass surrounding the excavation at mining. Mathematical modelling and analysis," in Proceedings of the AIP Conference Proceedings, vol. 1783, Tomsk, Russia, September 2016.

[31] C. Özgen Karacan, "Elastic and shear moduli of coal measure rocks derived from basic well logs using fractal statistics and radial basis functions," International Journal of Rock Mechanics \& Mining Sciences, vol. 46, no. 8, pp. 1281-1295, 2009.

[32] D. Li, E. Wang, X. Kong, M. Ali, and D. Wang, "Mechanical behaviors and acoustic emission fractal characteristics of coal specimens with a pre-existing flaw of various inclinations under uniaxial compression," International Journal of Rock Mechanics and Mining Sciences, vol. 116, pp. 38-51, 2019.

[33] L. Dexing, W. Enyuan, K. Xiangguo et al., "Fractal characteristics of acoustic emissions from coal under multi-stage true-triaxial compression," Journal of Geophysics and Engineering, vol. 15, no. 5, pp. 2021-2032, 2018.

[34] X. Ding, X. Xiao, X. Lv, D. Wu, and J. Xu, "Mechanical properties of bump-prone coal with different porosities and its acoustic emission-charge induction characteristics under uniaxial compression," Advances in Civil Engineering, vol. 2019, Article ID 7581061, 18 pages, 2019.

[35] X. Ding, X.-C. Xiao, D. Wu, and X.-F. Lv, "Mechanical properties and charge signal characteristics in coal material failure under different loading paths," International Journal of Coal Science \& Technology, vol. 6, no. 1, pp. 138-149, 2019.

[36] J. Swevers, F. Al-Bender, C. G. Ganseman, and T. Projogo, "An integrated friction model structure with improved presliding behavior for accurate friction compensation," IEEE Transactions on Automatic Control, vol. 45, no. 4, pp. 675686, 2000.

[37] T. H. Lee, K. K. Tan, and S. Huang, "Adaptive friction compensation with a dynamical friction model," IEEE/ASME Transactions on Mechatronics, vol. 16, no. 1, pp. 133-140, 2011.

[38] R. P. Nachane, G. F. S. Hussain, and K. P. K. Iyer, "Theory of stick-slip effect in friction," Indian Journal of Fribre \& Textile Research, vol. 23, pp. 201-208, 1998.

[39] R. Budakian and S. J. Putterman, "Correlation between charge transfer and stick-slip friction at a metal-insulator interface," Physical Review Letters, vol. 85, no. 5, pp. 1000-1003, 2000. 